\title{
How can I analyze large-scale intrinsic functional networks with iEEG?
}

\author{
Aaron Kucyi ${ }^{1}$, Sepideh Sadaghiani ${ }^{2}$
}

1. Department of Psychology, Northeastern University, Boston, MA

2. Psychology Department, University of Illinois at Urbana-Champaign, Urbana, IL

Note: This chapter is forthcoming in Intracranial EEG for Cognitive Neuroscience.

\begin{abstract}
$\underline{\text { Abstract }}$
An intrinsic functional brain network is a set of discrete, spatial elements that exhibit statistically dependent activity ("functional connectivity") with each other in a largely state-invariant manner (e.g. across wakeful rest, task performance, and sleep). Large-scale intrinsic networks - involving coupling between distant brain regions-were initially discovered with human functional neuroimaging (fMRI) based on hemodynamic signals. Though fMRI studies suggest critical relevance of these networks to brain function, findings remain challenging to interpret given the low temporal resolution and indirect nature of fMRI. Human iEEG is poised as a unique method that can deliver fundamental insights into the neurophysiological connectivity processes in intrinsic networks. In this chapter, we review iEEG analysis methods that have been used to identify electrophysiological networks closely resembling those found using classical fMRI functional connectivity. We focus on amplitude and phase coupling within multiple frequency bands as measures of iEEG intrinsic connectivity. We review evidence that iEEG connectivity shows state-invariant patterns of inter-regional coupling across multiple contexts. Moreover, we review applications of intrinsic iEEG connectivity patterns in predicting the roles of discrete neuronal populations in cognitive function. Finally, we explore how iEEG sheds light on the cognitive relevance of temporal dynamics within and between intrinsic networks.
\end{abstract}




\section{Introduction}

Cognitive neuroscience has classically emphasized the use of experimenteradministered task paradigms that actively engage targeted mental processes such as memory, attention, and decision-making. However, the brain exhibits highly coordinated patterns of spontaneous activity, even in the absence of explicit engagement in cognitive tasks or changes in external input. In a landmark finding with functional Magnetic Resonance Imaging (fMRI), researchers discovered that infraslow $(<0.1 \mathrm{~Hz})$ blood oxygenation level-dependent (BOLD) signals are highly correlated between distant regions in the brain when people are wakeful "at rest" (i.e., letting their minds wander freely) [1]. The spatial patterns of statistically dependent activity ("functional connectivity") in resting state fMRI reflect functional network integration at a large-scale level (i.e., coupling between distinct regions that are each typically $1 \mathrm{~mm}^{3}$ or larger). These large-scale fMRI networks partly (but not exclusively) reflect underlying anatomical connectivity [2] such as the coupling between homotopic regions of the two hemispheres that are structurally connected via commissural fibers [3]. The networks identified at rest also strongly resemble the most common patterns of brain-wide co-activation that are evoked during performance of cognitive tasks [4]. Strikingly, the same spatial architecture of functional connectivity observed at rest is also largely preserved during a wide variety of conditions, including sleep [5], anesthesia-induced unconsciousness[6, 7], and engagement in a diverse variety of cognitive tasks $[8,9]$. Thus, the state-invariant nature of these networks suggest a persistent role in "intrinsic" brain functions that cannot be attributed to immediate inputs or outputs $[10,11]$.

Since their discovery, there has been enormous interest within neuroscience in investigating the significance of large-scale intrinsic networks to healthy cognitive function, clinical dysfunction, neurodevelopment, and aging [12]. Moreover, there is increasing interest in the temporal dynamics of these networks in relation to ongoing cognition [13, 14]. However, findings remain challenging to interpret given the low temporal resolution, low signal-to-noise ratio, and indirect nature of fMRI, which relies on hemodynamic changes that reflect complex interactions of underlying neural and metabolic activity [15]. Non-invasive techniques, including scalp EEG and magnetoencephalography (MEG), have offered some insight into the electrophysiological basis of large-scale intrinsic networks [16]. Yet those techniques also suffer from low signal-to-noise as well as limited ability to precisely localize the anatomical sources of activity.

Thus, within human neuroscience, iEEG is poised as a unique method that can deliver fundamental insights into the neurophysiology of large-scale intrinsic networks and their dynamics. Directly implanted electrodes offer a combination of high signal-to-noise (higher than fMRI and EEG/MEG), high temporal resolution (higher than fMRI and similar to EEG/MEG), and spatial resolution (similar to fMRI and higher than EEG/MEG). Although iEEG cannot offer whole-brain coverage due to clinical considerations, activity is typically sampled from multiple locations distributed throughout the brain, often including simultaneous recording from distinct nodes within one or multiple intrinsic networks. Another unique aspect of iEEG, relative to most non-invasive methods, is that recordings are semi-chronic. This permits detailed investigations of intrinsic networks 
across various conditions and states (e.g. experimental tasks, naturalistic behavior, sleep) at multiple time scales.

In this chapter, we review iEEG analysis methods that have been used to identify and interrogate large-scale intrinsic networks. Given the rich information available in iEEG signals, multiple approaches can be used to investigate these networks. We focus primarily on coupling of iEEG signals within multiple frequency bands in terms of both amplitude and phase, two analysis methods that capture distinct neurophysiological processes (see also Chapter 36) $[17,18]$. After a brief overview of methodological considerations, we review evidence that iEEG connectivity shows state-invariant patterns of inter-regional coupling across multiple mental contexts. Moreover, we describe how iEEG intrinsic connectivity patterns predict task-evoked electrophysiological responses as well as the consequences of intracranial stimulation. Finally, we explore how iEEG sheds light on the functional significance of temporal dynamics within and between intrinsic networks.

\section{Methodological Considerations}

How are large-scale intrinsic networks typically analyzed in iEEG data? In contrast to $\mathrm{fMRI}$, it is usually not appropriate to examine iEEG intrinsic networks based on simply the raw signals, as these signals contain a broad range of frequency information with dissociable components. The spectral content of iEEG, when characterized as power as a function of frequency $f$, is non-uniform in that it shows a $1 / f$ pattern where low frequencies contribute disproportionately to the raw signal. As such, time-frequency decomposition is typically applied to isolate specific frequency bands prior to analyses of intrinsic network coupling [19]. There are various methodological options for performing time-frequency decomposition (e.g. Hilbert transform, wavelet-based methods), yet results have been largely concordant across studies of $\mathrm{iEEG}$ intrinsic networks regardless of the method choice [20-22]. Importantly, although time-frequency analysis methods decompose the data into distinct frequency bands, they do not guarantee that the derived time series contain oscillations (i.e., periodic/cyclical properties). Indeed, engagement of neuronal populations as measured with iEEG, either when evoked [23, 24] or spontaneous [25], is often characterized by a shift in broadband, non-oscillatory components of power amplitude. Recently, it has been suggested that band-limited oscillatory signals can be further disentangled from broadband $1 / f$-like fluctuations using dedicated tools (see also Chapter 24) $[26,27]$, but the potential value of such approaches to intrinsic network analyses has not yet been investigated.

Intrinsic networks are typically defined based on coupling of amplitude or phase of frequency band-limited activity across a time scale of seconds, minutes or longer (see Fig. 1 for overview). Amplitude coupling considers region pairs for which the strength (spectral power) of regional frequency band-limited activity waxes and wanes in an associated manner as being functionally connected. To estimate amplitude coupling, following rereferencing and minimal preprocessing of the raw iEEG data (see Chapter 27), timefrequency decomposition is first applied to each electrode signal (Fig. 1A-B). The decomposition can either be applied to an entire, continuous recording session of minutes or hours (a more computationally intensive approach) or to shorter time windows (e.g. 
sliding windows of seconds). Connectivity estimates from such short time windows can later be averaged to construct a 'static' connectivity organization, or alternatively can be employed to study time-varying dynamics in connectivity (cf. dedicated section below). After applying time-frequency analysis, estimates of the amplitude of band-limited power are obtained at each sample (termed "power amplitude" or "envelope") (Fig. 1C). At this stage, the continuous power amplitudes (or multiple estimates within shorter windows) from two different electrode locations can be correlated with one another to estimate functional connectivity in terms of amplitude coupling.

The major alternative family of within-frequency functional connectivity measures, phase coupling, is conceptualized as consistent phase relationships across brain regions. During periods of consistent phase difference (lag) of a given frequency across a pair of regions, the exchange of neural information is thought to be temporally well-aligned and efficient [28-30]. Many measures of phase coupling first estimate the time course of the phase of a particular band-limited oscillation. To this end, the Hilbert transform can be applied to the band-pass filtered signal time course at each electrode (the Wavelet transform offers an alternative). One common measure of phase lag consistency, the Phase Locking Value (PLV), is based on the stability (i.e., inverse of the variance) of a set of instantaneous phase differences [31]. In the original PLV formulation for task-based and trial-locked connectivity, the set of phase pairs either spans across trials at one given time point or across time points in individual trials. Adopted to studies of intrinsic connectivity, this set comes from consecutive phase cycles within a given time window over several seconds (to permit sufficient oscillation cycles $[18,32]$ ). Conversely, the Phase Lag Index (PLI) calculates the average of the sign of the phase difference over a given set of instantaneous phases [33] (see [34] for a more stable weighted version). An alternative and widely used approach to phase coupling is coherence (and variations thereof, e.g. [35]), which calculates cross-correlation in the frequency domain for a pair of electrodes [36]. Note however, that this measure does not only reflect interregional coupling of phases, but also amplitudes. Thus, coherence may be an appropriate choice if one conceptualizes the functional importance of phase synchrony to depend on the strength of the carrier oscillation. The choice of the particular measure of phase coupling therefore depends on the mechanistic view under consideration. Further, the different measures have different levels of susceptibility to volume conduction (imaginary coherence [35], imaginary PLV [32], and PLI/weighted PLI [33, 34] are thought to be less affected by this artifact; see below).

For completeness, we also note that beyond the within-frequency, band-limited measures most commonly employed and covered above, other approaches have also been explored to study intrinsic networks in iEEG. For example, instead of relying on spectral power or phase, intrinsic networks have been reported in cross-region correlations of the direct ECoG signal time courses in the infraslow range $(<0.5 \mathrm{~Hz})$ [37] and in canonical frequency bands [38]. Further, large-scale intrinsic network organization has been observed for cross-frequency coupling, where high-frequency broadband (HFB; 70-180 $\mathrm{Hz}$ ) in specific distributed regions is coupled to the phase of a slower oscillation in a seed region (see also Chapter 38) [39]. In light of the breadth of possible procedures to calculate 
electrophysiological long-range connectivity, we recommend careful deliberation of biological mechanisms of connectivity for a well-informed approach [16].

Important to the study of intrinsic networks, band-limited power amplitude time series and phase time courses contain frequencies that are much faster than BOLD fMRI signals. As such, when the goal is to investigate the electrophysiological connectivity processes that correspond to those known from fMRI, then temporal filtering is sometimes applied to the region-wise power amplitude estimates so that low frequencies can be isolated prior to computing inter-region correlations [40, 41]. Alternatively, one might be interested in the slow fluctuation range of the cross-region connectivity time course (when connectivity is calculated in a windowed, time-resolved manner). In this case, the time course of amplitude coupling or phase coupling can be temporally low-pass filtered (e.g. [42]). The temporal filtering method must be carefully considered [43]. When applied, distinct temporal filters are often explored and are commonly split into $>1 \mathrm{~Hz}, 0.1-1 \mathrm{~Hz}$, and $<0.1$ ranges $[21,22,41,44]$. The $<0.1 \mathrm{~Hz}$ range closely matches the infraslow component at which intrinsic BOLD networks are typically investigated, but this approach involves severe loss of higher frequency information in the estimates of power amplitude or cross-region phase- and amplitude coupling.

Using either filtered or unfiltered measures, a "seed-based" connectivity map can be generated for the coupling of one electrode to all other electrodes (Fig. 1D), or more globally, an 'all-to-all' connectivity matrix can be computed between all implanted electrode sites (Fig. 3A) [18]. Connectivity maps may be generated for multiple carrier frequencies, which can yield different patterns of results (Fig. 1E).
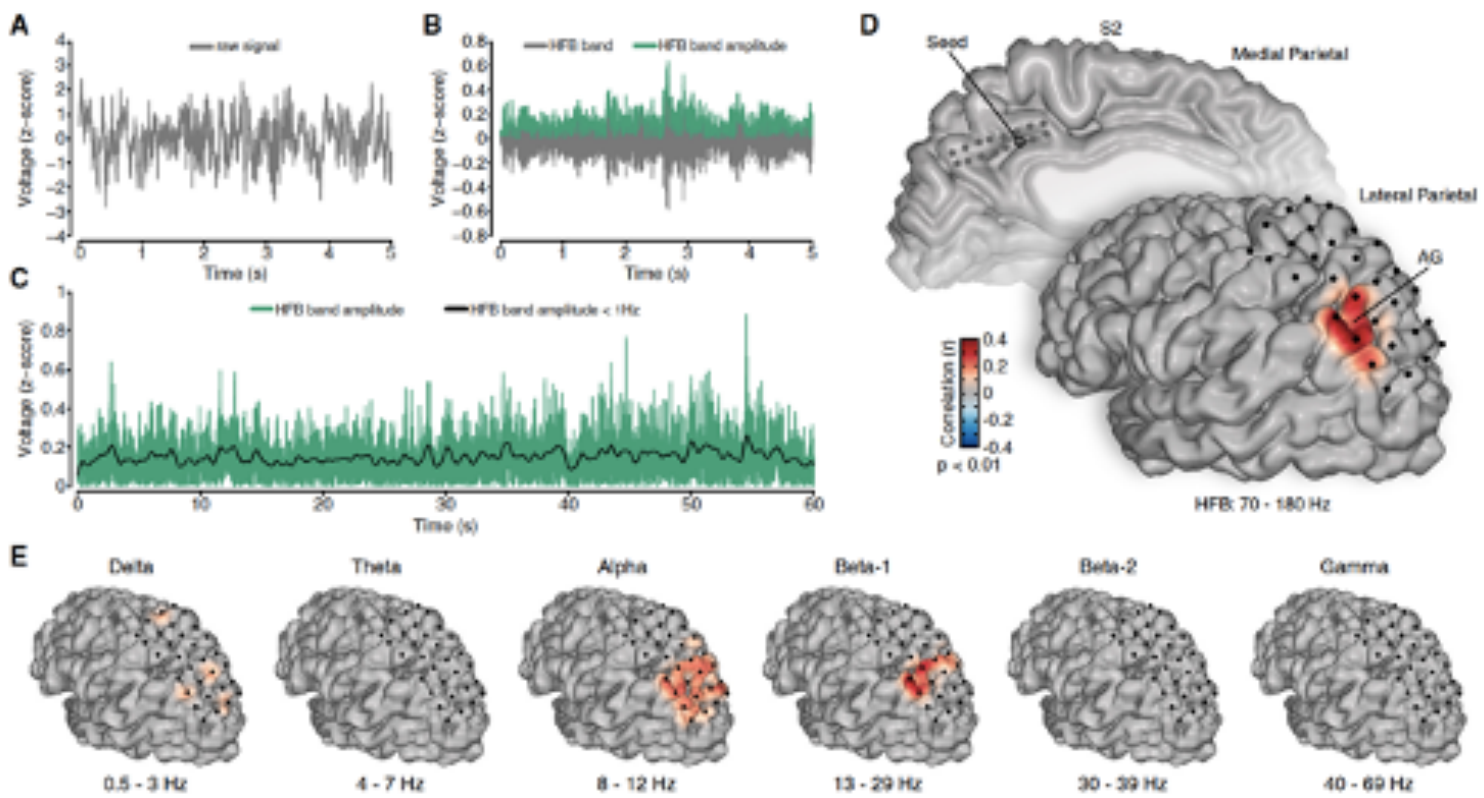

Figure 1. Illustration of how iEEG functional connectivity can be computed based on frequency band-limited power amplitude coupling. (A) Raw time series from a single electrode. (B) Filtered electrode time series in the high-frequency broadband (HFB; 70 $180 \mathrm{~Hz}$; gray) and HFB amplitude (green). (C) Unfiltered (green) and $<1 \mathrm{~Hz}$ filtered (black) HFB amplitude. (D) Seed-based correlation between a region in medial parietal 
cortex and other electrode sites (based on $<1 \mathrm{~Hz}$ filtered HFB amplitude). (E) Same as (D) but for a range of lower frequency bands. Adapted with permission from [44].

We close our methodological considerations with a note on accounting for physical distance. In amplitude-, phase-, and phase-amplitude coupling connectivity analyses, iEEG correlations between electrode sites are systematically greater at shorter, relative to longer, distances. There are multiple possible reasons for this relationship. On one hand, electrodes that are spatially proximal to one another may sample from distinct neuronal populations that are within the same network, thereby leading to genuine estimates of strong coupling. On the other hand, adjacent electrodes may partially sample from the same neuronal populations due to volume conduction or instantaneous field spread (see Chapter 19). Such factors has been well-described within the context of non-invasive EEG/MEG and can create artifactual connectivity estimates [45]. The problem is less severe in iEEG due to much greater proximity between each electrode and the neuronal population that it is sampling from [46]. However, for improved interpretability, studies of intrinsic network coupling have typically accounted for physical distance in their analyses. For example, when comparing the similarity of spatial maps of BOLD and iEEG functional connectivity, or between iEEG maps obtained across different states, physical distance (typically Euclidean distance) has been regressed out via partial correlation or cubic spline regression [20-22, 37, 47]. When spatial similarities remain consistent even after accounting for distance, the identified intrinsic network patterns are likely to be genuine rather than a product of volume conduction.

\section{Intrinsic Networks from Amplitude Coupling}

To date, most iEEG studies on large-scale intrinsic networks have focused on coupling of power amplitude within frequency band-limited ranges, typically involving within-frequency band coupling between distinct electrode sites. This focus builds on a rich literature of cognitive task iEEG studies, where stimulus-/task-evoked changes (relative to a baseline prestimulus state) in regional frequency-specific power amplitude have been found across a broad variety of conditions $[48,49]$. More recently, the selforganizing properties of task-independent, ongoing co-fluctuations in band-limited power amplitude have been investigated between regions across large-scale intrinsic networks $[18,20-22,37,41,42]$. Though a large range of carrier frequencies have been explored, much of this work has emphasized HFB or "high gamma" power amplitude, given that this component of the iEEG signal is known to correlate positively with the BOLD signal [50, 51] and with local neuronal population spiking rate near an iEEG electrode [52-54].

Using the above-described power amplitude connectivity analyses, researchers performed iEEG studies that confirmed an electrophysiological basis for large-scale intrinsic networks. Landmark studies by Nir et al. [41] and He et al. [37] provided initial evidence for neuroanatomically selective iEEG coupling patterns that resemble those originally identified with BOLD fMRI. A hallmark feature of intrinsic BOLD networks is inter-hemispheric coupling between homotopic regions [1,3]. Analyzing bihemispheric iEEG recordings from right and left auditory cortex, Nir et al. [41] investigated this interhemispheric phenomenon across resting state, task, and sleep states via correlations of 
band-limited power amplitudes across a broad range of frequency bands within the 1-100 $\mathrm{Hz}$ range. They found that across all states, gamma band-limited power $(40-100 \mathrm{~Hz})$ showed the strongest inter-hemispheric correlation, although significant coupling was also found at lower frequencies. When the infraslow $(<0.1 \mathrm{~Hz})$ component of gamma power amplitude was examined via temporal filtering, strong inter-hemispheric correlations $(\mathrm{r}=0.5$ to 0.8$)$ were found that approached the magnitude of correlations typically identified in fMRI data. This inter-hemispheric coupling was highly anatomically selective as it did not generalize to electrodes outside of the bilateral auditory regions. However, fMRI data were not available for direct comparison with iEEG. In complementary work, He et al. [37] more directly compared BOLD (obtained via pre- or post-surgical scans) versus ECoG functional connectivity of intrinsic networks in somatosensory, motor and surrounding regions. When investigating gamma $(50-100 \mathrm{~Hz})$ band-limited power, they found significant positive spatial correlations between BOLD and ECoG connectivity estimates, with some dependence on the state in which ECoG data were acquired (correlations were significant for waking data and REM sleep but not for slow-wave sleep). Together, these two studies [37, 41] provided the foundations for further work investigating intrinsic networks via iEEG power amplitude coupling across diverse behavioral states and broader brain systems.

Using similar power amplitude coupling analyses, iEEG intrinsic networks have now been identified within various systems extending well beyond auditory and sensorimotor regions, suggesting a general electrophysiological basis of intrinsic BOLD functional connectivity across individuals, brain networks, and behavioral states [21]. For example, iEEG has revealed intrinsic electrophysiological coupling across rest, task and sleep states within the default mode network (DMN) [55], one of the most frequently investigated intrinsic networks in neuroimaging research (Fig. 2). Anatomically-precise intrinsic coupling between nodes of the DMN has consistently been found based on fluctuations in HFB/high gamma power amplitude [20-22, 44]. Lower frequency ranges also show DMN organization that may be less anatomically specific [20,21, 44], though future work is needed to quantify the potential inter-frequency differences in spatial patterns within networks. Additionally, electrodes implanted within other well-described cortical association networks, such as the "dorsal attention" (DAN) and "frontoparietal control" networks, have consistently revealed intrinsic connectivity within the HFB power range [20, 21]. It was further suggested that these networks can be distinguished from one another based on preferential coupling within low-frequency bands (e.g. 4-8 Hz theta in DMN vs. 8-12 alpha in DAN) [20], a phenomenon that requires more in-depth future study. The state-invariant nature of intrinsic iEEG networks across wider systems has also been confirmed; similarities in spatial organization have been repeatedly observed across rest, task and sleep states [21, 44, 47].

In addition to within-network coupling, the power amplitude approach has also been used to examine the electrophysiology of between-network phenomena. A major finding that emerged from intrinsic connectivity fMRI is a negative correlation (anticorrelation) between signals in the DMN and other networks, especially DAN and salience networks [56-58]. During active task performance, the DMN typically exhibits stimulus- or task-evoked BOLD deactivation when the DAN and salience network show 
BOLD activation, an inter-network pattern often referred to as 'functional antagonism' [59]. This phenomenon has been extensively validated with iEEG based on task-evoked HFB power amplitude [60-62]. However, whether or not intrinsic (state-invariant) anticorrelation, including presence during a resting state, is genuinely grounded in neurophysiological dynamics has been a matter of debate due to technical limitations of fMRI [63]. Within this context, iEEG has been used to investigate the neurophysiology of intrinsic DMN anticorrelation. In short duration ( 3-6 minute) resting state iEEG recordings, Keller et al. [22] reported DMN anticorrelations within the low-frequency $(<1$ $\mathrm{Hz}$ ) component of high-frequency broadband $(50-150 \mathrm{~Hz})$ power amplitude fluctuations. However, when comparing resting state iEEG to BOLD correlations (using presurgical fMRI), only a subset of negative BOLD correlations corresponded to negative iEEG correlations. Moreover, iEEG anticorrelations were generally weaker than those found in BOLD data [22].

Importantly, however, power amplitude connectivity analysis methods typically assume zero-lag relationships between regions. Task-evoked iEEG responses have revealed that the onset of activations within the DAN and salience network precedes the onset of DMN deactivation by hundreds of milliseconds [61, 62], a phenomenon that fMRI has been blind to. Thus, Kucyi et al. [62] investigated lagged iEEG interactions between neuronal populations that were functionally localized within the DMN, DAN or salience network based on task-evoked response profiles. Using a lagged cross-correlation of HFB power amplitude, they found DMN anticorrelations in both rest and task conditions. However, relative to rest, the magnitude of these lagged anticorrelations was stronger during a continuous performance task that required sustained attention [62]. Future iEEG studies may shed light on the rapid, temporally dynamic processes that may underlie DMN anticorrelations as well as broader, intrinsic coordination between networks. Methodologically, this work highlights limitations of power amplitude coupling analyses that purely rely on zero-lag relationships, suggesting a need for future studies to account for lagged inter-regional relationships. Important contributions of lagged relationships to intrinsic network organization have been recently demonstrated based on slow fluctuations in fMRI and iEEG signals [64], but iEEG can also be leveraged to generate much more precise, millisecond-level insights.

A

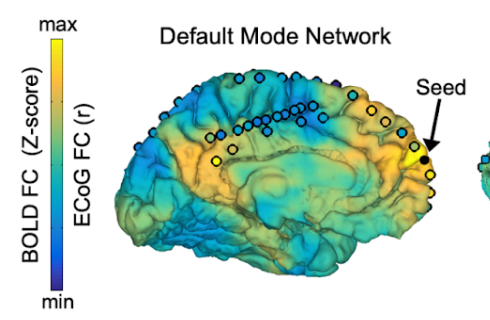

Resting State Correlations

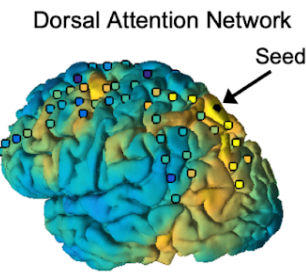

B Sleep Correlations (Default Mode Network)

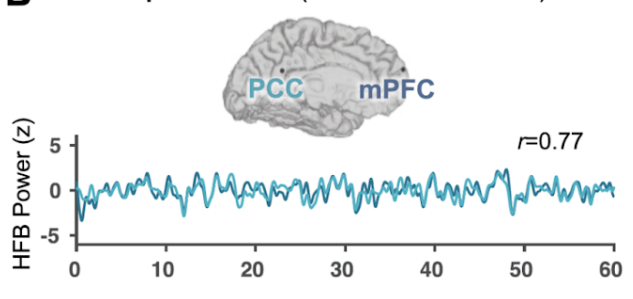

Figure 2. Power amplitude coupling between core regions within major intrinsic networks originally described with fMRI. (A) Correspondence between presurgical resting state BOLD functional connectivity (shown on cortical surface) and ECoG high-frequency broadband (70-170 Hz; 0.1-1 Hz filtered envelope) functional connectivity within the same patient's brain (fMRI and ECoG data acquired separately). The medial prefrontal ( $\mathrm{PPFC}$ ) 
and posterior cingulate (PCC) cortices, within the default mode network (left), show strong, anatomically selective coupling in both modalities. The superior parietal lobule and frontal eyes fields, within the dorsal attention network (right), similarly show strong selective coupling. (B) Highly correlated fluctuations (high-frequency broadband power amplitude; 0.1-1 Hz filtered) between $\mathrm{mPFC}$ and PCC persists during sleep (in same patient as shown in (A)). Adapted with permission from [21].

\section{Intrinsic Networks from Phase Coupling}

While the majority of iEEG investigations of large-scale intrinsic networks have focused on amplitude coupling as discussed above, phase coupling approaches have delivered a network organization that is spatially convergent with that of amplitude coupling. Given this alignment, the current section provides only a brief discussion of some of the major phase coupling-based findings.

The stark mechanistic difference between amplitude coupling and phase coupling (see methodological considerations above; [17]) invites the question of how their intrinsic spatial organization relates to each other. Mostame and Sadaghiani [18] quantified the spatial similarity of ECoG-derived connectivity matrices across the two connectivity modes during short periods (1.5 or $2.5 \mathrm{~s})$ of pre-stimulus baseline and post-stimulus task processing (finger flexing and verb generation). The study investigated stimulus-related changes from pre-stimulus to post-stimulus periods, as well as intrinsic connectivity present during both periods. The study confirmed that phase- and amplitude coupling constitute distinct connectivity processes, since stimulus-related changes in the two connectivity modes occurred in different connections and were temporally independent. Importantly however, when assessing the distributed pattern of intrinsic connectivity, amplitude correlations showed a spatial pattern substantially similar to that of phase coupling during both post- and pre-stimulus periods. This observation held true irrespective of the choice of phase coupling measure (PLV, weighted PLI and imaginary coherence). The stability of the shared, distributed intrinsic network organization across baseline and active (post-stimulus) periods further suggests that this organization may be largely invariant to cognitive contexts.

Indeed, direct investigations of connectivity patterns over various cognitive contexts confirms such state invariance. Kramer et al. [65] measured frequency-specific coherence in day-long ECoG recordings comprising various cognitive states. They estimated the core intrinsic organization from static connectivity over the full $\sim 24-\mathrm{hr}$ recording. They then demonstrated that this core organization is observable in shorter recordings $(>=100 \mathrm{~s})$ during a broad range of mental states consisting of wakefulness, drowsiness, as well as stage II and III sleep. In experimentally controlled task states and resting wakefulness, Mostame and Sadaghiani [47] directly compared spatial connectivity patterns between cognitive contexts. They found strong spatial resemblance of phase coupling during the active trial (post-stimulus) period of various tasks, task-free, prestimulus baseline, and task-free resting state in all frequency bands (Fig. 3B). Of note, a substantial part of the intrinsic connectivity pattern of individual frequency bands was spatially shared across frequencies (Fig. 3C). Equivalent results were observed for 
amplitude coupling. Collectively, these findings show that long-range electrophysiological connectivity is governed by a spatial organization that is relatively stable over mental and cognitive states, largely shared across the breadth of canonical oscillatory bands, and comparable across phase coupling and amplitude coupling.

A. Singe-subject example of connectivity matrices
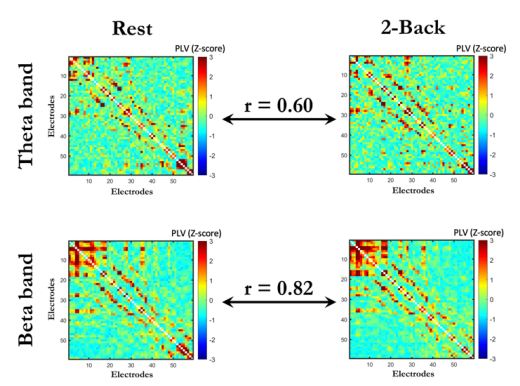

B. Spatial correspondence across cognitive states

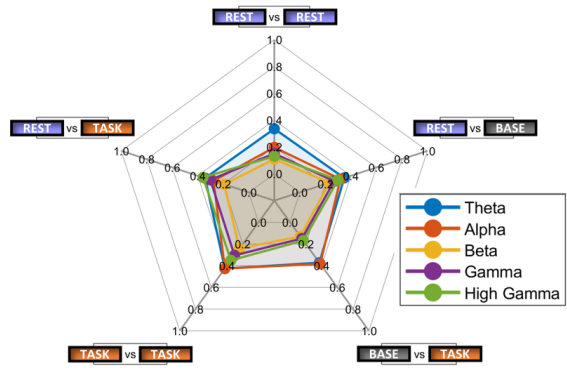

C. Spatial correspondence across frequency bands

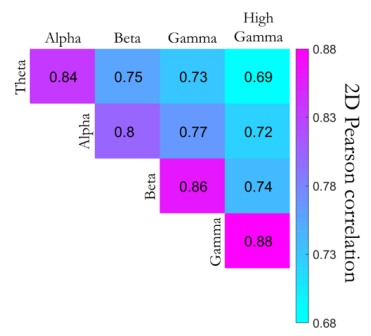

Figure 3. Invariance of the intrinsic spatial organization of electrophysiological connectivity over cognitive states and oscillatory frequency bands. (A) All-to-all electrode phase coupling matrices for an example subject. For both the theta (top row) and beta band (bottom row), the connectivity pattern showed strong spatial correlation between task-free wakefulness (left) and a cognitively demanding working memory 2-back task (right). A (weaker) similarity can also be visually appreciated between the frequency bands (top vs. bottom). (B) Spatial correlation was assessed for a group of subjects between the connectivity matrices from resting wakefulness ("rest"), pre-stimulus baseline ("base"), and active trial-related processing during the post-stimulus period of various different tasks ("task"). Group-average values are shown for the cross-state dissimilarity (1 minus spatial correlation). In all frequency bands, this dissimilarity was no greater across mental states than between two resting state periods. $(\boldsymbol{C})$ The group-average spatial correlations across frequency-specific connectivity patterns (consensus over mental states) showed strong spatial correlation between frequency bands. All visualized data are derived using Phase locking Value (PLV) on ECoG data. Equivalent results were observed for bandspecific amplitude correlations. Adapted with permission from [47].

\section{Applications to the Study of Cognition}

Once intrinsic networks are identified, how can they inform or predict the role of neuronal populations in cognitive functions? In this section, we cover evidence from two key types of applications that highlight the value of iEEG intrinsic connectivity to the study of cognition: (A) Predicting task-evoked electrophysiological responses; and (B) Predicting experiential effects elicited by intracranial stimulation.

First, iEEG studies have demonstrated that intrinsic connectivity is tightly related to the tendency of two electrode sites to co-activate with each other in response to a cognitive task or external stimulus [44, 66]. Predictions of task-evoked iEEG responses can be generated from intrinsic connectivity as estimated from the same individual's presurgical resting state fMRI [67] or from a population-level atlas of intrinsic networks 
(e.g. [68]) when fMRI data are not available (though the latter approach may be less accurate). Additionally, intrinsic connectivity (e.g. during a resting state) can be estimated directly from iEEG to predict task-evoked responses. For example, Foster et al. [44] showed that two key nodes of the DMN (posteromedial cortex and angular gyrus) responded with increased HFB power to a task condition involving autobiographical memory recall. The same sites showed strong, anatomically-selective coupling of slow $(<1$ $\mathrm{Hz}$ filtered) fluctuations in HFB power during resting and sleep states. In another example, Kucyi et al. [62] showed that task-evoked HFB response profiles could be predicted based on the location of a given electrode within the DMN, DAN or salience network (as predefined based on fMRI-based intrinsic network atlas) (Fig. 4). Such evidence aligns more generally with prior fMRI work, where it has been shown that at the global (wholebrain) level, regions exhibiting functional connectivity also tend to co-activate with one another during cognitive task performance $[4,69]$. The reason that intrinsic networks tend to resemble task-evoked responses remains under debate, and future iEEG studies may shed light on the neurophysiological mechanisms that underlie such relationships. For example, it is possible that spontaneous activity is constrained by statistical histories of coactivation [70], provides predictive signals for perception [11, 71], and partly reflects ongoing experiences and cognitive processes [14].

Second, intrinsic connectivity can predict how intracranial stimulation will cause a change in a patient's subjective experience or behavior $[72,73]$. High-frequency (typically $50-100 \mathrm{~Hz}$ ) stimulation is often applied in the iEEG setting for the clinical purpose of functional mapping (see also Chapters 4 and 39). Importantly, the intrinsic network identity that an electrode is located within can determine whether or not an effect of stimulation will be found and what the specific experiential or behavioral qualities of an effect will be. Indeed, the classical behavioral effects that are evoked by intracranial stimulation of sensorimotor regions have been used to validate fMRI intrinsic connectivity parcellation methods [74]. In an application to more complex cognitive function, Parvizi et al. [72] showed that stimulation of electrodes implanted within the mid-cingulate cortex-when specifically localized to the salience network-evoked the subjective, narrative experience within patients that was termed by the experimenters a "will to persevere." Furthermore, in a larger whole-cortex analysis across 67 patients, Fox et al. [75] showed that stimulation of a given electrode within the DMN, compared to stimulation of other networks, is less likely to result in any experiential or behavioral response. However, it was recently shown that intracranial stimulation of DMN regions (localized with presurgical fMRI intrinsic connectivity) affects performance in a creative thinking task, even though patients may not be aware of stimulation-evoked changes in subjective experience or behavior [76].

Taken together, these findings strongly highlight the relevance of intrinsic connectivity to the study of cognitive function with iEEG. Additionally, these findings may have important practical implications for research and clinical procedures conducted within the iEEG setting. For example, they raise the possibility that intrinsic functional connectivity, which can be estimated during relatively passive conditions (e.g. resting state, sleep), may serve as a substitute for, or initial screening prior to, cognitive task performance or electrical stimulation. Given that some cognitive tasks are highly demanding or difficult for patients to perform, and that electrical stimulation procedures can involve patient 
burden, intrinsic connectivity estimates can play an important role in determining which tasks should be administered or which electrodes should be stimulated in a given patient.
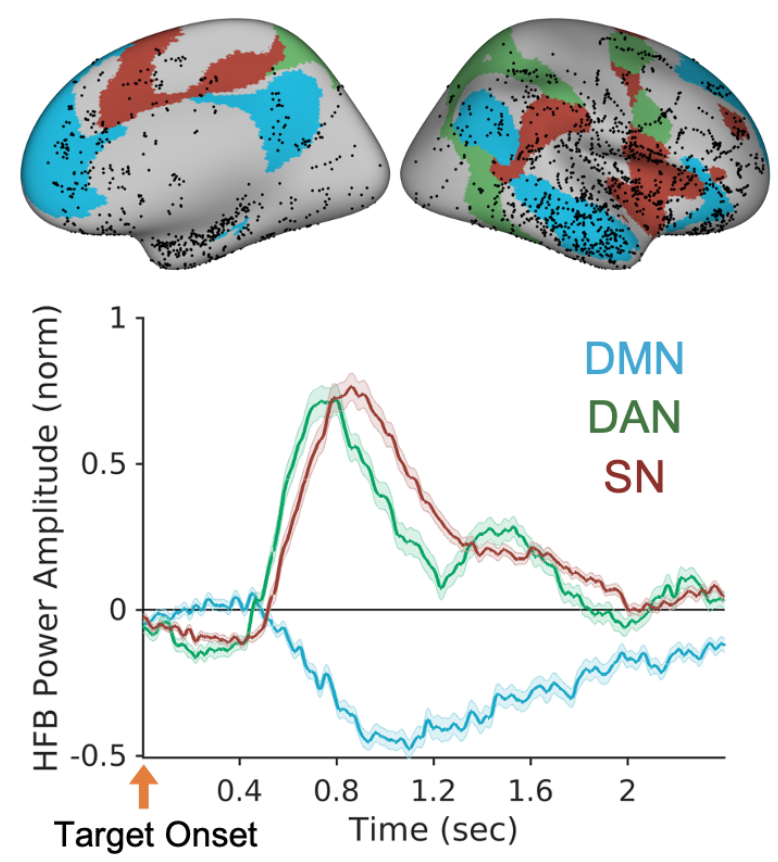

Figure 4. Intrinsic network organization predicts task-evoked electrophysiological HFB response profile. (Top) Locations of electrodes implanted within cortical nodes of the default mode network (DMN; blue), dorsal attention network (DAN; green), and salience network (SN; red) in a cohort of 31 patients. (Bottom) Average task-evoked HFB response profiles of electrode sites that showed a significant response to a visual target stimulus that appeared in $\sim 10 \%$ trials in a continuous performance task. Following target onset, increased HFB activation appears earlier in DAN than in $S N$ sites, and DMN deactivations are later and more sustained than the activations in both other networks. Adapted with permission from [62] (Creative Commons license: https://creativecommons.org/licenses/by/4.0/).

\section{Temporal Dynamics of Intrinsic Networks}

This chapter has so far considered intrinsic connectivity as 'static' in that the described analysis approaches and applications explicitly search for iEEG coupling that is stable across different states. However, though intrinsic network architecture is largely state-invariant, changes occur in the strength of coupling over time [65, 77]. Such connectivity changes can be externally elicited but can also occur spontaneously. These fluctuations are often subtle yet significant, suggesting that intrinsic activity 'explores' a dynamic functional repertoire of distinct brain states about a central core organization [78]. Time-varying functional connectivity analysis has recently risen in popularity in resting state fMRI [79], with some findings beginning to highlight how ongoing network fluctuations relate to spontaneous cognitive and affective processes, including arousal, attention, memory replay and mind wandering (see also Chapter 30) [14, 80]. Yet those findings are limited due to poor fMRI temporal resolution that cannot capture activity 
relevant to cognitive events that may arise on the time scale of milliseconds. It is thus within this area in the study of intrinsic networks-their temporal dynamics-where iEEG may have potential to offer its most unique scientific insights into the nature of cognition. The study of intrinsic network dynamics with iEEG is still in its infancy. We here briefly highlight select examples of preliminary insights, and point to future research directions, on the cognitive and behavioral relevance of iEEG network dynamics.

The spontaneous dynamics of iEEG networks at 'rest,' and their behavioral relevance, have been studied across multiple time scales [25, 65, 81, 82]. Importantly, ongoing changes in inter-electrode coupling, or regional activity at electrodes localized to intrinsic networks, have been related to changes in external variables that reflect ongoing cognition. For example, time-varying iEEG amygdalar and hippocampal network configurations across distinct 15-20 minute resting state windows were predictive of selfreported mood fluctuations $[83,84]$. This suggests that even at relatively slow time scales, intrinsic network connectivity is not fully stable and that its changes across time windowseven lasting tens of minutes-meaningfully relate to subjective experiences that underlie mood states. Future iEEG studies that employ experience sampling may shed more light on the relevant time scales of network dynamics that correspond to richer varieties of ongoing conscious phenomena.

At a faster time scale, Kucyi and Parvizi [25] showed that spontaneous, transient increases in HFB power amplitude ("activations") during a resting state were often of similar magnitude to task-evoked activations within a dorsal anterior insular cortex (daIC) region localized to the salience network. Moreover, both task-evoked and spontaneous daIC activations reliably preceded pupillary dilations by several hundred milliseconds, suggesting a role in autonomic arousal regardless of whether it is externally-elicited or selfgenerated [25]. Future work is needed to examine how these spontaneous, functionally significant, daIC events are dynamically coupled to wider brain networks to mediate the ongoing changes in arousal and cognition that naturally arise "at rest."

Another emerging area of importance in the study of temporal dynamics of intrinsic networks concerns the role of "sharp-wave ripple" (SWR) events that occur spontaneously in the hippocampus (see Chapter 30). These robust neuronal synchronization events have been well characterized in animal models [85] and can be detected in the human brain uniquely using iEEG [86-89]. Hippocampal SWRs occur more frequently during resting states than during active task performance and are hypothesized to function in the consolidation, and spontaneous reactivation, of memory [85]. The dialogue between hippocampal SWRs and large-scale intrinsic network dynamics in the human brain, and its potential significance to ongoing cognition, remains poorly characterized. However, it was recently shown that during cued autobiographical memory recollection, hippocampal ripples occur in concert with DMN activation [90]. Further iEEG studies of intrinsic activity (e.g. during rest and 'naturalistic' states involving spontaneously generated behavior) may further reveal the potentially critical role of hippocampal SWRs in coordinating intrinsic brain network dynamics on multiple time scales. 


\section{Conclusion}

In this chapter, we reviewed iEEG analysis methods for studying large-scale intrinsic brain networks and described how these approaches are playing an increasingly important role in the study of human cognition. To date, iEEG has been critical in validating evidence from other neuroimaging modalities for the existence of large-scale intrinsic networks. The convergence of evidence with that from other modalities and populations (e.g. neurotypical individuals) supports the idea that $\mathrm{EEEG-despite} \mathrm{its} \mathrm{usual}$ reliance on patients with epilepsy-provides meaningful insights into the nature of typical intrinsic brain organization. Critically, beyond simply validating other modalities, iEEG can deliver unique insights into the temporal dynamics of intrinsic networks that go well beyond what can be gleaned from other human neuroscience techniques. Future iEEG studies are expected to yield fundamental insights into how the brain dynamically selforganizes itself to support cognition. 


\section{$\underline{\text { References }}$}

1. Biswal B, Yetkin FZ, Haughton VM, Hyde JS (1995) Functional connectivity in the motor cortex of resting human brain using echo-planar MRI. Magn Reson Med 34:537-541.

2. Honey CJ, Sporns O, Cammoun L, et al (2009) Predicting human resting-state functional connectivity from structural connectivity. Proc Natl Acad Sci U S A 106:2035-2040.

3. Lowe MJ, Mock BJ, Sorenson JA (1998) Functional connectivity in single and multislice echoplanar imaging using resting-state fluctuations. Neuroimage 7:119_ 132.

4. Smith SM, Fox PT, Miller KL, et al (2009) Correspondence of the brain's functional architecture during activation and rest. Proc Natl Acad Sci U S A 106:13040-13045.

5. Fukunaga M, Horovitz SG, van Gelderen P, et al (2006) Large-amplitude, spatially correlated fluctuations in BOLD fMRI signals during extended rest and early sleep stages. Magn Reson Imaging 24:979-992.

6. Peltier SJ, Kerssens C, Hamann SB, et al (2005) Functional connectivity changes with concentration of sevoflurane anesthesia. Neuroreport 16:285-288.

7. Vincent JL, Patel GH, Fox MD, et al (2007) Intrinsic functional architecture in the anaesthetized monkey brain. Nature 447:83-86.

8. Gratton C, Laumann TO, Nielsen AN, et al (2018) Functional Brain Networks Are Dominated by Stable Group and Individual Factors, Not Cognitive or Daily Variation. Neuron 98:439-452.e5.

9. Cole MW, Bassett DS, Power JD, et al (2014) Intrinsic and task-evoked network architectures of the human brain. Neuron 83:238-251.

10. Fox MD, Raichle ME (2007) Spontaneous fluctuations in brain activity observed with functional magnetic resonance imaging. Nat Rev Neurosci 8:700-711.

11. Sadaghiani S, Kleinschmidt A (2013) Functional interactions between intrinsic brain activity and behavior. Neuroimage 80:379-386.

12. Zhang J, Kucyi A, Raya J, et al (2021) What have we really learned from functional connectivity in clinical populations? Neuroimage 242:118466.

13. Hutchison RM, Womelsdorf T, Allen EA, et al (2013) Dynamic functional connectivity: promise, issues, and interpretations. Neuroimage 80:360-378. 
14. Kucyi A, Tambini A, Sadaghiani S, et al (2018) Spontaneous cognitive processes and the behavioral validation of time-varying brain connectivity. Netw Neurosci 2:397417.

15. Logothetis NK (2008) What we can do and what we cannot do with fMRI. Nature 453:869-878.

16. Sadaghiani S, Brookes MJ, Baillet S (2021) Connectomics of human electrophysiology. Neuroimage 247:118788.

17. Engel AK, Gerloff C, Hilgetag CC, Nolte G (2013) Intrinsic coupling modes: multiscale interactions in ongoing brain activity. Neuron 80:867-886.

18. Mostame P, Sadaghiani S (2020) Phase- and amplitude-coupling are tied by an intrinsic spatial organization but show divergent stimulus-related changes. Neuroimage 219:117051.

19. Foster BL, He BJ, Honey CJ, et al (2016) Spontaneous Neural Dynamics and Multiscale Network Organization. Front Syst Neurosci 10:7.

20. Hacker CD, Snyder AZ, Pahwa M, et al (2017) Frequency-specific electrophysiologic correlates of resting state fMRI networks. Neuroimage 149:446-457.

21. Kucyi A, Schrouff J, Bickel S, et al (2018) Intracranial Electrophysiology Reveals Reproducible Intrinsic Functional Connectivity within Human Brain Networks. J Neurosci 38:4230-4242.

22. Keller CJ, Bickel S, Honey CJ, et al (2013) Neurophysiological investigation of spontaneous correlated and anticorrelated fluctuations of the BOLD signal. J Neurosci 33:6333-6342.

23. Miller KJ, Leuthardt EC, Schalk G, et al (2007) Spectral changes in cortical surface potentials during motor movement. J Neurosci 27:2424-2432.

24. Ramot M, Fisch L, Harel M, et al (2012) A widely distributed spectral signature of task-negative electrocorticography responses revealed during a visuomotor task in the human cortex. J Neurosci 32:10458-10469.

25. Kucyi A, Parvizi J (2020) Pupillary Dynamics Link Spontaneous and Task-Evoked Activations Recorded Directly from Human Insula. J Neurosci 40:6207-6218.

26. Wen H, Liu Z (2016) Separating Fractal and Oscillatory Components in the Power Spectrum of Neurophysiological Signal. Brain Topogr 29:13-26.

27. Donoghue T, Haller M, Peterson EJ, et al (2020) Parameterizing neural power spectra into periodic and aperiodic components. Nat Neurosci 23:1655-1665. 
28. Varela F, Lachaux JP, Rodriguez E, Martinerie J (2001) The brainweb: phase synchronization and large-scale integration. Nat Rev Neurosci 2:229-239.

29. Singer W (1999) Neuronal synchrony: a versatile code for the definition of relations? Neuron 24:49-65, 111-125.

30. Fries P (2005) A mechanism for cognitive dynamics: neuronal communication through neuronal coherence. Trends Cogn Sci 9:474-480.

31. Lachaux JP, Rodriguez E, Martinerie J, Varela FJ (1999) Measuring phase synchrony in brain signals. Hum Brain Mapp 8:194-208.

32. Sadaghiani S, Scheeringa R, Lehongre K, et al (2012) $\alpha$-band phase synchrony is related to activity in the fronto-parietal adaptive control network. J Neurosci 32:14305-14310.

33. Stam CJ, Nolte G, Daffertshofer A (2007) Phase lag index: assessment of functional connectivity from multi channel EEG and MEG with diminished bias from common sources. Hum Brain Mapp 28:1178-1193.

34. Vinck M, Oostenveld R, van Wingerden M, et al (2011) An improved index of phasesynchronization for electrophysiological data in the presence of volume-conduction, noise and sample-size bias. Neuroimage 55:1548-1565.

35. Nolte G, Bai O, Wheaton L, et al (2004) Identifying true brain interaction from EEG data using the imaginary part of coherency. Clin Neurophysiol 115:2292-2307.

36. Wang J, Tao A, Anderson WS, et al (2021) Mesoscopic physiological interactions in the human brain reveal small-world properties. Cell Rep 36:109585.

37. He BJ, Snyder AZ, Zempel JM, et al (2008) Electrophysiological correlates of the brain's intrinsic large-scale functional architecture. Proc Natl Acad Sci U S A 105:16039-16044.

38. Betzel RF, Medaglia JD, Kahn AE, et al (2019) Structural, geometric and genetic factors predict interregional brain connectivity patterns probed by electrocorticography. Nat Biomed Eng 3:902-916.

39. Weaver KE, Wander JD, Ko AL, et al (2016) Directional patterns of cross frequency phase and amplitude coupling within the resting state mimic patterns of fMRI functional connectivity. Neuroimage 128:238-251.

40. Leopold DA, Murayama Y, Logothetis NK (2003) Very slow activity fluctuations in monkey visual cortex: implications for functional brain imaging. Cereb Cortex 13:422-433. 
41. Nir Y, Mukamel R, Dinstein I, et al (2008) Interhemispheric correlations of slow spontaneous neuronal fluctuations revealed in human sensory cortex. Nat Neurosci $11: 1100-1108$.

42. Ko AL, Weaver KE, Hakimian S, Ojemann JG (2013) Identifying functional networks using endogenous connectivity in gamma band electrocorticography. Brain Connect 3:491-502.

43. de Cheveigné A, Nelken I (2019) Filters: When, Why, and How (Not) to Use Them. Neuron 102:280-293.

44. Foster BL, Rangarajan V, Shirer WR, Parvizi J (2015) Intrinsic and task-dependent coupling of neuronal population activity in human parietal cortex. Neuron 86:578590.

45. Palva JM, Wang SH, Palva S, et al (2018) Ghost interactions in MEG/EEG source space: A note of caution on inter-areal coupling measures. Neuroimage 173:632643.

46. Dubey A, Ray S (2019) Cortical Electrocorticogram (ECoG) Is a Local Signal. J Neurosci 39:4299-4311.

47. Mostame P, Sadaghiani S (2021) Oscillation-Based Connectivity Architecture Is Dominated by an Intrinsic Spatial Organization, Not Cognitive State or Frequency. J Neurosci 41:179-192.

48. Crone NE, Miglioretti DL, Gordon B, et al (1998) Functional mapping of human sensorimotor cortex with electrocorticographic spectral analysis. I. Alpha and beta event-related desynchronization. Brain 121 ( Pt 12):2271-2299.

49. Lachaux J-P, Axmacher N, Mormann F, et al (2012) High-frequency neural activity and human cognition: past, present and possible future of intracranial EEG research. Prog Neurobiol 98:279-301.

50. Mukamel R, Gelbard H, Arieli A, et al (2005) Coupling between neuronal firing, field potentials, and FMRI in human auditory cortex. Science 309:951-954.

51. Hermes D, Miller KJ, Vansteensel MJ, et al (2012) Neurophysiologic correlates of fMRI in human motor cortex. Hum Brain Mapp 33:1689-1699.

52. Manning JR, Jacobs J, Fried I, Kahana MJ (2009) Broadband shifts in local field potential power spectra are correlated with single-neuron spiking in humans. J Neurosci 29:13613-13620.

53. Ray S, Maunsell JHR (2011) Different origins of gamma rhythm and high-gamma activity in macaque visual cortex. PLoS Biol 9:e1000610. 
54. Nir Y, Fisch L, Mukamel R, et al (2007) Coupling between neuronal firing rate, gamma LFP, and BOLD fMRI is related to interneuronal correlations. Curr Biol $17: 1275-1285$.

55. Fox KCR, Foster BL, Kucyi A, et al (2018) Intracranial Electrophysiology of the Human Default Network. Trends Cogn Sci 22:307-324.

56. Fox MD, Snyder AZ, Vincent JL, et al (2005) The human brain is intrinsically organized into dynamic, anticorrelated functional networks. Proc Natl Acad Sci U S A 102:9673-9678.

57. Fransson P (2005) Spontaneous low-frequency BOLD signal fluctuations: an fMRI investigation of the resting-state default mode of brain function hypothesis. Hum Brain Mapp 26:15-29.

58. Chai XJ, Castañón AN, Öngür D, Whitfield-Gabrieli S (2012) Anticorrelations in resting state networks without global signal regression. NeuroImage 59:1420-1428.

59. Buckner RL, DiNicola LM (2019) The brain's default network: updated anatomy, physiology and evolving insights. Nat Rev Neurosci 20:593-608.

60. Ossandón T, Jerbi K, Vidal JR, et al (2011) Transient suppression of broadband gamma power in the default-mode network is correlated with task complexity and subject performance. J Neurosci 31:14521-14530.

61. Raccah O, Daitch AL, Kucyi A, Parvizi J (2018) Direct Cortical Recordings Suggest Temporal Order of Task-Evoked Responses in Human Dorsal Attention and Default Networks. J Neurosci 38:10305-10313.

62. Kucyi A, Daitch A, Raccah O, et al (2020) Electrophysiological dynamics of antagonistic brain networks reflect attentional fluctuations. Nat Commun 11:325.

63. Murphy K, Fox MD (2017) Towards a consensus regarding global signal regression for resting state functional connectivity MRI. Neuroimage 154:169-173.

64. Mitra A, Snyder AZ, Hacker CD, et al (2016) Human cortical-hippocampal dialogue in wake and slow-wave sleep. Proc Natl Acad Sci U S A 113:E6868-E6876.

65. Kramer MA, Eden UT, Lepage KQ, et al (2011) Emergence of persistent networks in long-term intracranial EEG recordings. J Neurosci 31:15757-15767.

66. Daitch AL, Foster BL, Schrouff J, et al (2016) Mapping human temporal and parietal neuronal population activity and functional coupling during mathematical cognition. Proc Natl Acad Sci U S A 113:E7277-E7286.

67. Dastjerdi M, Foster BL, Nasrullah S, et al (2011) Differential electrophysiological response during rest, self-referential, and non-self-referential tasks in human posteromedial cortex. Proc Natl Acad Sci U S A 108:3023-3028. 
68. Yeo BTT, Krienen FM, Sepulcre J, et al (2011) The organization of the human cerebral cortex estimated by intrinsic functional connectivity. J Neurophysiol

69. Tavor I, Parker Jones O, Mars RB, et al (2016) Task-free MRI predicts individual differences in brain activity during task performance. Science 352:216-220.

70. Harmelech T, Malach R (2013) Neurocognitive biases and the patterns of spontaneous correlations in the human cortex. Trends Cogn Sci 17:606-615.

71. Pezzulo G, Zorzi M, Corbetta M (2021) The secret life of predictive brains: what's spontaneous activity for? Trends Cogn Sci 25:730-743.

72. Parvizi J, Rangarajan V, Shirer WR, et al (2013) The will to persevere induced by electrical stimulation of the human cingulate gyrus. Neuron 80:1359-1367.

73. Parvizi J, Braga RM, Kucyi A, et al (2021) Altered sense of self during seizures in the posteromedial cortex. Proc Natl Acad Sci U S A 118:e2100522118.

74. Wang D, Buckner RL, Fox MD, et al (2015) Parcellating cortical functional networks in individuals. Nat Neurosci 18:1853-1860.

75. Fox KCR, Shi L, Baek S, et al (2020) Intrinsic network architecture predicts the effects elicited by intracranial electrical stimulation of the human brain. Nat Hum Behav 4:1039-1052.

76. Shofty B, Gonen T, Bergmann E, et al (2022) The default network is causally linked to creative thinking. Mol Psychiatry 1-7.

77. Chang C, Glover GH (2010) Time-frequency dynamics of resting-state brain connectivity measured with fMRI. Neuroimage 50:81-98.

78. Kringelbach ML, Deco G (2020) Brain States and Transitions: Insights from Computational Neuroscience. Cell Rep 32:108128.

79. Lurie DJ, Kessler D, Bassett DS, et al (2020) Questions and controversies in the study of time-varying functional connectivity in resting fMRI. Netw Neurosci 4:30_ 69.

80. Martin CG, He BJ, Chang C (2021) State-related neural influences on fMRI connectivity estimation. Neuroimage 244:118590.

81. Casimo K, Madhyastha TM, Ko AL, et al (2019) Spontaneous Variation in Electrocorticographic Resting-State Connectivity. Brain Connect 9:488-499.

82. Breshears JD, Hamilton LS, Chang EF (2018) Spontaneous Neural Activity in the Superior Temporal Gyrus Recapitulates Tuning for Speech Features. Front Hum Neurosci 12:360. 
83. Kirkby LA, Luongo FJ, Lee MB, et al (2018) An Amygdala-Hippocampus Subnetwork that Encodes Variation in Human Mood. Cell 175:1688-1700.e14.

84. Scangos KW, Khambhati AN, Daly PM, et al (2021) Closed-loop neuromodulation in an individual with treatment-resistant depression. Nat Med.

85. Buzsáki G (2015) Hippocampal sharp wave-ripple: A cognitive biomarker for episodic memory and planning. Hippocampus 25:1073-1188.

86. Axmacher N, Elger CE, Fell J (2008) Ripples in the medial temporal lobe are relevant for human memory consolidation. Brain 131:1806-1817.

87. Helfrich RF, Lendner JD, Mander BA, et al (2019) Bidirectional prefrontalhippocampal dynamics organize information transfer during sleep in humans. Nat Commun 10:3572.

88. Norman Y, Yeagle EM, Khuvis S, et al (2019) Hippocampal sharp-wave ripples linked to visual episodic recollection in humans. Science 365:eaax1030.

89. Vaz AP, Inati SK, Brunel N, Zaghloul KA (2019) Coupled ripple oscillations between the medial temporal lobe and neocortex retrieve human memory. Science 363:975-978.

90. Norman Y, Raccah O, Liu S, et al (2021) Hippocampal ripples and their coordinated dialogue with the default mode network during recent and remote recollection. Neuron 109:2767-2780.e5. 